\title{
Short-course isoniazid plus rifapentine therapy for latent tuberculosis in Brazil
}

\author{
Julio Croda ${ }^{[1],[2]}$
}

[1]. Faculdade de Ciências da Saúde, Universidade Federal da Grande Dourados, Dourados, Mato Grosso do Sul, Brazil. [2]. Fundação Oswaldo Cruz, Campo Grande, Mato Grosso do Sul, Brazil.

Brazil is experiencing an epidemiological transition, wherein the incidence of tuberculosis has significantly decreased over the last few years. The World Health Organization's (WHO) new objective for tuberculosis control is to reduce the incidence to below 10 per 100,000 inhabitants by $2035^{(1)}$. To achieve this target, we must ensure that early diagnosis and care is made available to people in poor communities, people living with human immunodeficiency virus/acquired immunodeficiency syndrome (HIV/AIDS), the homeless, indigenous populations, illicit drug users, and prison populations, which currently comprise the majority of tuberculosis cases. Early diagnosis continues to be a priority in disease control; this has been reinforced by the Ministry of Health decision to extend the diagnostic network by implementing Xpert (Cepheid, Dx System Version 4.0c) machine nationwide. In this new context of disease elimination, one of the new priorities is the treatment of latent tuberculosis infection (LTBI).

A recently introduced regimen based on 12 doses of rifapentine with isoniazid has been evaluated. This regimen has been found to reduce the treatment time from 6 months to 3 months and the number of doses from 180 to $12^{(2)}$. A manuscript entitled Rifapentine for tuberculosis treatment infection latent in the general population and human immunodeficiency virus-positive patients: summary of evidence, which is published in the same issue of the Brazilian Society of Tropical Medicine $e^{(3)}$, provides an important review of the subject and justifies the recommendation of this new regimen for the treatment of LTBI in Brazil.

Two randomized clinical trials (RCTs) were reviewed. In both trials, the 12-dose regimen combining rifapentine and isoniazid for 3 months had the same effect as the traditional regimen of isoniazid alone, patient adherence was greater $\left(95.7 \%\right.$ versus $83.8 \%{ }^{(4)}$ and $82.1 \%$ versus $\left.69 \%{ }^{(5)}\right)$ (Figure 1), and hepatotoxicity was lower $\left(1.5 \%\right.$ versus $2.4 \%, \mathrm{p}>0.05^{(4)} ; 0.4 \%$ versus $2.7 \%, \mathrm{p}<0.001^{(5)}$ ) (Figure 1). Another recent clinical trial with 6862 participants confirmed these results and showed high adherence and low hepatotoxicity rates $(0.4 \%$ versus $1.8 \%$, $\mathrm{p}<0.01)^{(6)}$. Similar findings were observed in studies in children

Corresponding author: Dr. Julio Croda. Faculdade de Ciências da Saúde/UFGD. Rodovia Dourados-Itaum, km 12, Caixa Postal 364, 79804-970 Dourados, Mato Grosso do Sul, Brasil.

Phone: 5567 3410-2325; Fax: 5567 3410-2320

e-mail: juliocroda@ufgd.edu.br; julio.croda@fiocruz.br

Received 29 September 2015

Accepted 6 October 2015

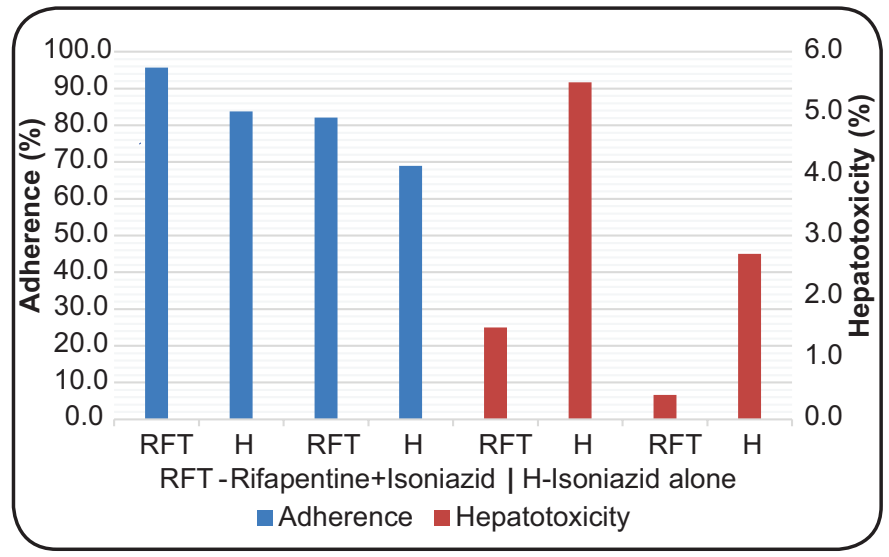

FIGURE 1 - Comparisons of adherence and hepatotoxicity between the two treatment regimens for latent tuberculosis infection.

2-17 years old, in people living with HIV/AIDS on antiretroviral therapy, and in organ transplantation patients ${ }^{(7)}{ }^{(8)}\left({ }^{(9)}\right.$. Costeffectiveness studies have demonstrated the reduced economic impact of this new regimen ${ }^{(10)(11)}$. A fixed-dose combination of rifapentine $(300 \mathrm{mg})$ and isoniazid $(300 \mathrm{mg})$ is expected to be marketed soon in tablet form, which will make treatment easier.

To reach the next WHO milestone, LTBI treatment needs to be expanded in Brazil. In the context of a new, simplified short-course regimen, we expect better adherence and coverage of directly observed treatment. In addition to these new recommendations, to increase the diagnosis and treatment of LTBI in Brazil, it is necessary to invest in national manufacturing to provide reagents for diagnosing LTBI and maintaining this new treatment regimen.

\section{CONFLICT OF INTEREST}

The author declare that there is no conflict of interest.

\section{REFERENCES}

1. Uplekar M, Weil D, Lonnroth K, Jaramillo E, Lienhardt C, Dias HM, et al. WHO's new end TB strategy. Lancet 2015; 385: 1799-1801. 
2. Sharma SK, Sharma A, Kadhiravan T, Tharyan P. Rifamycins (rifampicin, rifabutin and rifapentine) compared to isoniazid for preventing tuberculosis in HIV-negative people at risk of active TB. Cochrane Database Syst Rev 2013; 7:CD007545.

3. Vidal JS, Silva MT, Sanchez MN. Rifapentine for latent tuberculosis infection treatment in the general population and human immunodeficiency virus-positive patients: summary of evidence. Rev Soc Bras Med Trop 2015; 48:507-513.

4. Martinson NA, Barnes GL, Moulton LH, Msandiwa R, Hausler H, Ram M, et al. New regimens to prevent tuberculosis in adults with HIV infection. N Engl J Med 2011; 365:11-20.

5. Sterling TR, Villarino ME, Borisov AS, Shang N, Gordin F, BlivenSizemore E, et al. Three months of rifapentine and isoniazid for latent tuberculosis infection. N Engl J Med 2011; 365:2155-2166.

6. Bliven-Sizemore EE, Sterling TR, Shang N, Benator D, Schwartzman K, Reves R, et al. Three months of weekly rifapentine plus isoniazid is less hepatotoxic than nine months of daily isoniazid for LTBI. Int J Tuberc Lung Dis 2015; 19:1039-1044.

7. Villarino ME, Scott NA, Weis SE, Weiner M, Conde MB, Jones $\mathrm{B}$, et al. Treatment for preventing tuberculosis in children and adolescents: a randomized clinical trial of a 3-month, 12-dose regimen of a combination of rifapentine and isoniazid. JAMA Pediatr 2015; 169: 247-255.

8. Podany AT, Bao Y, Swindells S, Chaisson RE, Andersen JW, Mwelase T, et al. Efavirenz Pharmacokinetics and Pharmacodynamics in HIV-Infected Persons Receiving Rifapentine and Isoniazid for Tuberculosis Prevention. Clin Infect Dis 2015; 61:1322-1327.

9. de Castilla DL, Rakita RM, Spitters CE, Narita M, Jain R, Limaye AP. Short-course isoniazid plus rifapentine directly observed therapy for latent tuberculosis in solid-organ transplant candidates. Transplant 2014; 97:206-211.

10. Frick M, Seaworth B, McKenna L, Elnour T, Lee C. Costeffectiveness of 12-dose LTBI regimen improved following advocacy to lower the price of rifapentine. Int J Tuberc Lung Dis 2014; $18: 1386$.

11. Shepardson D, Marks SM, Chesson H, Kerrigan A, Holland DP, Scott N, et al. Cost-effectiveness of a 12-dose regimen for treating latent tuberculous infection in the United States. Int J Tuberc Lung Dis 2013 ; 17:1531-1537. 